Revista Matemática Universitária, vol. 1, 2019

ISSN: 2675-5254-DOI: https ://doi .org/10.21711/26755254/rmu202011

\title{
PROPAGAÇÃO DE PACOTES DE ONDAS: BREATHERS
}

\author{
MIGUEL A. ALEJO
}

\begin{abstract}
Resumo. Neste artigo explicaremos como a natureza encontrou uma maneira de transportar ondas usando os chamados pacotes de ondas ou breathers, que são solução de algumas equações da Física Matemática.
\end{abstract}

\section{INTRODUÇÃO}

A observação de estruturas dinâmicas na natureza que mantêm sua forma enquanto evoluem ao longo do tempo (também chamadas de estruturas coerentes) é um fato permanente na história da humanidade. Por exemplo, lembre-se do formato de algumas nuvens, dos padrões nas folhas das plantas ou na vegetação, nos desenhos nas conchas de moluscos bivalves, nos minerais, e assim por diante. A curiosidade humana impulsionou o questionamento da origem de tais estruturas, procurando explicar e entender como elas surgem e como podem ser geradas. Para obter as respostas corretas para tais questões, a Matemática é a ferramenta chave, desenvolvendo os modelos matemáticos que descrevem as características principais dessas estruturas coerentes.

Focando particularmente em estruturas observadas na água, temos a pororoca (ou onda de maré, veja [23]) no Brasil, que é um fenômeno recorrente da interação dos rios pouco profundos com as marés atlânticas do norte e nordeste do país. O mais notável desse espectáculo

Data de aceitação: 22 de setembro de 2019.

Palavras chave. Propagação, pacote, breather, onda.

* M.A. Alejo teve apoio da Bolsa de Prod. CNPq nu. 305205/2016-1.

O autor deseja destacar os valiosos comentarios e sugestões por parte dos pareceristas, que melhorarem de maneira significativa uma versão anterior deste artigo. Gostaria também agradecer aos professores Adán J. Corcho (IM-UFRJ), Didier Pilod (UiBergen), Eliezer Batista e Matheus C. Bortolan (UFSC) pelas discussões e observações críticas feitas ao texto. 
da natureza é a presença de uma onda que, mantendo seu perfil ao longo de grandes distâncias (documentadas em até $50 \mathrm{~km}$ rio adentro), é arrastada pela maré nos dias de lua cheia, e se dirige em contrafluxo rio adentro, elevando o caudal do rio e arrasando as margens e tudo o que encontra em seu caminho.

Embora esse fenômeno já fosse conhecido há séculos no Brasil e na América do Sul (no inicio do século XVI, na região norte do Brasil, a pororoca quase afundou a caravela do pioneiro navegante espanhol Vicente Yáñez Pinzón), a primeira observação documentada de uma estrutura coerente na água, evoluindo no tempo, foi do engenheiro escocês John Scott Russell ${ }^{1}$ em 1834 (ver [21]). O que ele observou exatamente enquanto passeava a cavalo, paralelamente a um canal de água rasa, que era utilizado para transporte de mercadorias em pequenos barcos puxados por cavalos de ambos os lados, foi o seguinte, nas suas próprias palavras:

I was observing the motion of a boat which was rapidly drawn along a narrow channel by a pair of horses, when the boat suddenly stopped -not so the mass of water in the channel which it had put in motion; it accumulated round the prow of the vessel in a state of violent agitation, then suddenly leaving it behind, rolled forward with great velocity, assuming the form of a large solitary elevation, a rounded, smooth and well-defined heap of water, which continued its course along the channel apparently without change of form or diminution of speed. I followed it on horseback, and overtook it still rolling on at a rate of some eight or nine miles an hour, preserving its original figure some thirty feet long and a foot to a foot and a half in height. Its height gradually diminished, and after a chase of one or two miles I lost it in the windings of the channel. Such, in the month of August 1834, was my first chance interview with that singular and beautiful phenomenon which I have called the Wave of Translation...

Resumindo, o que Russell observou em 1834 foi uma onda (que ele chamou de onda de translação) em um canal de água rasa, ou pouco funda, produzida pela inércia do barco, a qual se propagou, sem perder sua forma e sua altura, ao longo do canal por uma distância considerável. Esse fenômeno de propagação chamou tanto a atenção de Russell que ele tentou explicá-la usando alguns modelos matemáticos, e tentou também recriá-la em seu laboratório, propondo uma fórmula para a velocidade de propagação da onda no final da sua pesquisa, dada por

$$
c^{2}=g(h+A)
$$

\footnotetext{
${ }^{1}$ John Scott Russell (Glasgow, 1808 - Ilha de Wight, 1882) foi empossado como professor na Universidade de Edimburgo aos vinte e quatro anos e membro da Royal Society. Foi também um exitoso engenheiro naval, desenhou a primeira fragata encoraçada (o Warrior) e o primeiro barco à vapor de grandes dimensões, sendo de fato o maior barco do mundo até início do século XX.
} 
onde $g$ é a aceleração da gravidade, $h$ a altura da camada de água em estado de repouso e $A$ a amplitude da onda. Observe que em (1), quanto maior a amplitude $A$ da onda, maior velocidade $c$ ela atinge. Ele também foi capaz de produzir em seu laboratório duas ondas simultâneas com diferentes alturas e afastando-se uma da outra.

Entretanto, embora intuitivamente se possa entender o termo dispersar, o quê significa exatamente ser dispersivo?

Um sistema dispersivo é aquele no qual a velocidade de grupo depende do número de ondas, isto é, são aqueles sistemas nos quais a velocidade de grupo não é constante. Mais precisamente, considerando soluções de tipo onda plana

$$
u(t, x) \approx e^{i(k x+\omega t)},
$$

o termo $\omega$ é a velocidade de fase e o termo $\omega^{\prime}(k)$ chama-se de velocidade de grupo ${ }^{2}$. Portanto, um sistema será dispersivo sempre que $\omega^{\prime \prime}(k) \neq 0$.

Por exemplo, as soluções da equação ${ }^{3}$

$$
u_{t}+u_{x}=0
$$

não são dispersivas (note que $\omega(k)=-k$ ), mas as soluções da equação de Airy

$$
u_{t}+u_{x x x}=0
$$

são dispersivas (note que $\omega(k)=k^{3}$ ). De fato, uma superposição contínua de ondas planas será a solução de (4)

$$
u(t, x)=\int_{\mathbb{R}} e^{i\left(k x+k^{3} t\right)} d k .
$$

De fato, para $t \neq 0$, a integral anterior pode ser calculada explicitamente $^{4}$ em termos da função de Airy $\operatorname{Ai}(x)=u(1, x)$, isto é

$$
u(t, x)=\frac{1}{t^{1 / 3}} A i\left(\frac{x}{t^{1 / 3}}\right), \quad x \in \mathbb{R}, t>0 .
$$

Pode se demostrar que a função de Airy $A i$ é regular e decai a 0 no infinito. Além disso, $A i(x)$ é solução da equação diferencial

$$
\frac{d^{2} y}{d x^{2}}-x y=0
$$

Portanto, as soluções de (4) vão diminuindo sua amplitude $u$ e se dispersam á medida que o tempo $t$ aumenta.

Obviamente, a equação de Airy (4) não pode descrever o comportamento de ondas solitárias descrito por Russell, pois suas soluções

\footnotetext{
${ }^{2}$ Aqui ' significa a derivada com respeito ao argumento.

${ }^{3}$ Aqui $u_{t} \equiv \frac{\partial u}{\partial t} \equiv \partial_{t} u$ e $u_{x} \equiv \frac{\partial u}{\partial x} \equiv \partial_{x} u$.

${ }^{4}$ Observar que devido ás grandes oscilações no infinito causadas pela não linearidade $k^{3}$ na variável de integração, temos convergência e por tanto podemos calculá-la explicitamente.
} 
dispersam. É preciso então um outro modelo. Depois dessas primeiras tentativas, outros engenheiros e cientistas tentaram melhorar a explicação para obter soluções mais parecidas e semelhantes ao fenômeno observado, sendo que o modelo seguinte (simples e unidimensional), proposto em 1895, e chamado de KdV (por seus criadores, Diederik Korteweg e Gustav de $\left.\operatorname{Vries}^{5}[14]\right)$

$$
v_{t}+v_{x x x}+v v_{x}=0
$$

foi o primeiro modelo ${ }^{6}$ a gerar de maneira matemática ondas solitárias dentre as suas soluções. Em (7), v é a altura da perturbação na camada de água do canal de água rasa.

A equação de $\mathrm{KdV}$ (7) satisfaz várias invariâncias, que permitem ampliar o número de soluções encontradas. Por exemplo, se $v(t, x)$ é solução de $(7)$, então $\frac{1}{\mu} v\left(\frac{t}{\sqrt{\mu^{3}}}, \frac{x}{\sqrt{\mu}}\right), \quad \mu \in \mathbb{R}^{+}$(transformação de escala) e $\mu+v(t, x-\mu t), \quad \mu \in \mathbb{R}$ (transformação de Galileu) são também soluções de (7). Esse último tipo de transformação permite "decolar" a solução, conseguindo descrever então a altura $\mu>0$ da camada de água sobre a qual se levanta a perturbação na forma de onda de translação, que Russell observou.

Voltando à (7), observe que se descartamos o termo não linear $v v_{x}$ em (7), a equação torna-se a equação de Airy (4), onde já vimos que as soluções dispersam. Portanto, o modelo KdV (7) diz-nos que é preciso a concorrência de termos dispersivos $\left(v_{t}+v_{x x x}\right)$ contra termos não lineares ou concentradores $\left(v v_{x}\right)$ para que, em um equilíbrio adequado, gerem soluções que não dispersam, mantendo sua forma no tempo. É o que se chamam soluções de tipo onda solitária.

Uma onda solitária, do ponto de vista matemático, pode ser considerada como uma solução da equação diferencial correspondente, da forma $f(x \pm c t)$, onde $f$ é uma função que satisfaz a equação diferencial, e $c$ é considerada a velocidade de propagação. Isto é, o perfil da função $f$ move-se para a esquerda ou direita (dependendo se tivermos $+c$ ou $-c$ na velocidade) ao longo do tempo.

No caso concreto da equação (7), as suas ondas solitárias são fáceis de obter. Propondo soluções do tipo $u(t, x)=f(x-c t)$ e substituindo

\footnotetext{
${ }^{5}$ Diederik Johannes Korteweg (1848-1941) iniciou os seus estudos de engenheria mas logo voltou para a Matemática, fazendo estudos de doutorado sob a orientação de Van der Waals e finalizando em 1878 com uma tese entitulada Sobre a propagação de ondas em um tubo elástico. Ele se tornou depois professor na Universidade de Amsterdã e teve como principal contribuição científica o trabalho [14] em $1895 \mathrm{em}$ conjunto com seu aluno de doutorado, Gustav de Vries (1866-1934).

${ }^{6}$ Em realidade, essa equação já apareceu anos antes, em 1877, num trabalho de Joseph Valentin Boussinesq [10], mas o trabalho que ficou conhecido por obter tais soluções em forma de onda solitária foi o de Korteweg-de Vries [14].
} 
em (7), obtemos a equação diferencial (aqui ' quer dizer derivada com respeito a $\xi=x-c t)$

$$
-c f^{\prime}+f^{\prime \prime \prime}+f f^{\prime}=0,
$$

e integrando em $\xi$ e levando em conta que procuramos soluções com a propriedade $f \underset{\xi \rightarrow \pm \infty}{\longrightarrow} 0$, obtemos a equação

$$
f^{\prime \prime}-c f+\frac{1}{2} f^{2}=0,
$$

que pode ser resolvida em termos de funções conhecidas. De fato as soluções de (9) (e portanto também soluções de (7)) são dadas pela forma funcional

$$
Q_{c}(t, x):=3 \operatorname{csech}^{2}\left(\frac{\sqrt{c}}{2}\left(x-c t+x_{0}\right)\right),
$$
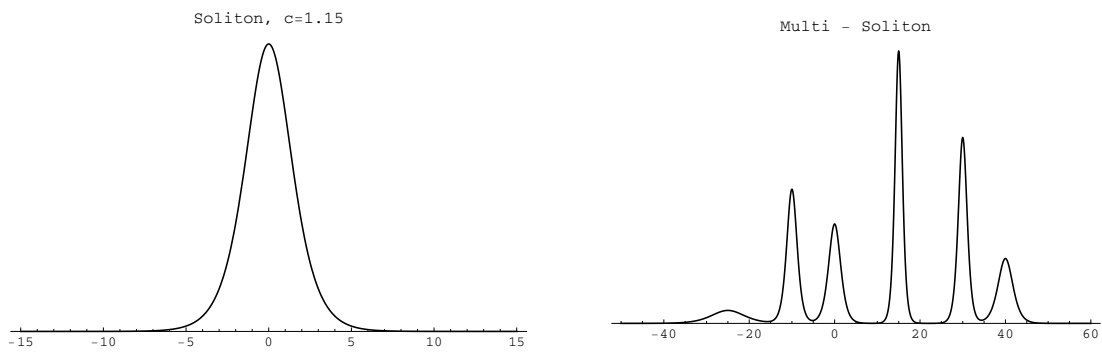

Figura 1. Esquerda: Sóliton (10) de KdV $\operatorname{com} c=$ 1.15 e $x_{0}=0$. Direita: Multi-Sóliton (11) obtido como superposição de 6 sólitons com diferentes scalings $c_{i}, i=$ $1, \ldots 6$.

com $x_{0} \in \mathbb{R}$ fixado de modo arbitrário. Voltando ás soluções (10), essas ondas de translação são chamadas de ondas solitárias, ou viajantes, ou também sólitons. Observe também que na solução (10) anterior, quanto maior a sua amplitude $c$, maior é a sua velocidade de propagação $c$, sendo essa uma propriedade típica das ondas, como bem sabem as pessoas que vivem do mar, e também os surfistas.

Obviamente, da mesma maneira que observamos na realidade uma única onda como (10), também podem ser observadas, em condições de águas rasas (por exemplo na beira de uma praia com inclinação pequena, num dia com pouco vento) duas, três ou mais ondas seguidas, propagando-se. Portanto, se o modelo matemático proposto for suficientemente bom, ele teria também que reproduzir ou replicar esses fenômenos de várias ondas consecutivas. Afortunadamente, o modelo KdV (7) reproduz essas multiondas ou $n$ ondas solitárias, da seguinte 
forma:

$R(t, x):=12 \frac{\partial^{2}}{\partial x^{2}} \log (F(t, x)) \approx \sum_{i=1}^{n}\left(Q_{c_{i}}\left(t, x-x_{i}\right)+\rho_{c_{i}}(t, x)\right), n \in \mathbb{N}$,

$\operatorname{com} F(t, x)$ uma certa função conhecida, dependendo das velocidades $c_{i}$ (ver [1], [16], [12] ou [18] para uma expressão explícita da mesma). De fato, como pode se ver no lado direito, essa expressão pode se aproximar como uma soma de ondas solitárias $Q_{c_{i}}(10)$ com diferentes velocidades $c_{i}$ e separadas por quantidades determinadas pelos $x_{i}$. Além disso, aqui $\rho_{c_{i}}(t, x)$ é um termo que se dissipa no tempo, indo para 0 . Observe que se em (8) supomos condições não nulas para $f$ nos extremos, isto é, se depois da integração obtivermos

$$
f^{\prime \prime}-c f+\frac{1}{2} f^{2}=a, \quad a \neq 0,
$$

então podemos obter soluções periódicas (no espaço) para a equação $(7)$.

Voltando ás multiondas (11), observe que como as ondas têm diferentes alturas $c_{i}$, e portanto velocidades $c_{i}$ diferentes, elas se afastam umas das outras, sendo difícil ter "controle" sobre elas. Bom, uma maneira de ter "controle" sobre as ondas seria escolher uma outra solução com todas as velocidades $c_{i}$ iguais a uma velocidade comum $c$, e separando cada uma das ondas, na mesma distância $x_{i} \equiv \bar{x}$, e assim ter todas as ondas indo na mesma velocidade e sem se afastar entre elas. Apesar desse estado poder ser construído analiticamente, é muito difícil observar tal distribuição na realidade, como consequência do grande número de fatores aleatórios que afetam a precisa colocação de uma tal distribuição ordenada inicialmente. Em certa medida, é um estado de ondas "artificial" e a natureza tende a não se comportar exatamente desse modo.

Como fazer então para "controlar" ou "empacotar" as ondas? Como a natureza faz? A resposta para essas perguntas são os chamados pacotes de ondas (um exemplo aparece na Figura 2) como veremos na próxima seção.

\section{PACOTES Simples}

Antes de tentar "controlar" ondas, como falávamos na seção anterior, é preciso entendê-las melhor. Assim, para abranger a descrição de mais fenômenos ondulatórios observados, ao longo do tempo foram propostas melhorias e generalizações do modelo (7). Por exemplo, Miura percebeu em [19], que através da transformação

$$
v(t, x):=\left(u^{2}+u_{x}\right)(t, x),
$$




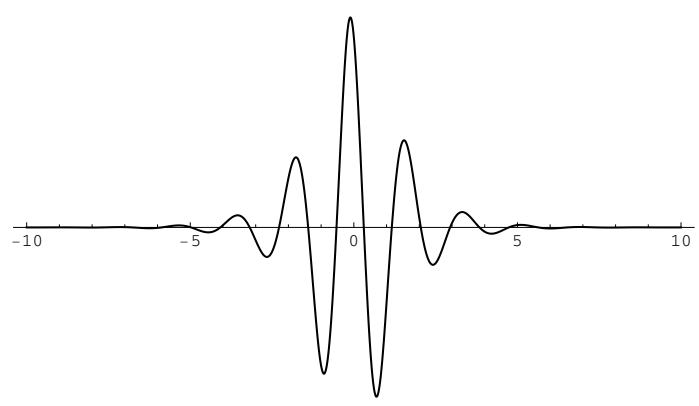

Figura 2. Um exemplo de pacote de onda no instante inicial $t=0$.

a equação KdV (7) torna-se outra equação, chamada de equação modificada de $\mathrm{KdV}$ (ou mKdV) desfocalizante (pelo sinal - na frente da não linearidade)

$$
u_{t}+u_{x x x}-u^{2} u_{x}=0
$$

ou no caso de trabalhar com a transformação complexa

$$
v(t, x):=\left(u^{2}+i u_{x}\right)(t, x),
$$

a $\mathrm{KdV}(7)$ converte-se na correspondente equação modificada de $\mathrm{KdV}$ (ou mKdV) focalizante (pelo sinal + na frente da não linearidade)

$$
u_{t}+u_{x x x}+u^{2} u_{x}=0 .
$$

Resumindo, o que Miura encontrou foram as chamadas transformações de Miura (13) e (15), que levam, como o leitor pode comprovar, soluções da KdV (7) em soluções da mKdV (14) e (16), dependendo da transformação usada.

Além do mais, pode-se estabelecer uma outra equação relacionada com a KdV (7) a partir da seguinte observação: procurando na $\mathrm{mKdV}$ focalizante (16) (por exemplo), soluções do tipo $u(t, x)=\mu+w(t, x)$ com $\mu \in \mathbb{R}$, e depois de fazer uma translação simples no espaço, pode-se encontrar simplesmente a equação de Gardner

$$
w_{t}+w_{x x x}+\mu w w_{x}+w^{2} w_{x}=0, \quad \mu \in \mathbb{R},
$$

que como pode ser visto, é uma mistura entre os modelos KdV (7) e o mKdV (16). Ambos os modelos (16) e (17) também descrevem propagação de ondas solitárias, tendo soluções explícitas que podem ser encontradas. Por exemplo, da mesma maneira que a (7), a solução sóliton de (16) é dada por

(18) $u(t, x)=Q_{c}\left(x-c t+x_{0}\right), \quad Q_{c}(s):=\sqrt{c} Q(\sqrt{c} s), \quad c>0, x_{0} \in \mathbb{R}$, onde $x_{0}$ é uma translação fixa de modo arbitrário e com $Q(x):=$ $\sqrt{6} \operatorname{sech}(x)$. De fato, $Q_{c}>0$ é solução da equação diferencial

$$
Q_{c}^{\prime \prime}-c Q_{c}+\frac{1}{3} Q_{c}^{3}=0,
$$


ou reescrita em termos de um operador diferencial de segunda ordem da seguinte forma

$$
\mathcal{T}_{c} Q_{c}=0, \quad \mathcal{T}_{c}:=\partial_{x}^{2}-c+\frac{1}{3} Q_{c}^{2}
$$

e que chamaremos de operador do sóliton. A mKdV (16) também tem soluções na forma de múltiplas ondas solitárias de maneira parecida ao exemplo de (11), sendo que uma das consequências diretas de ter essas multiondas, ou $N$ ondas solitárias, é que sob certas condições elas podem se acoplar e grudar como um todo. O exemplo mais simples aparece no caso de duas ondas solitárias. Para a mKdV (16) por exemplo, isto é quando $N=2 \mathrm{em}$ (11). Nesse caso particular, quando os parâmetros $c_{1}$ e $c_{2}$ estão relacionados pela conjugação complexa, assim $\sqrt{c_{1}}=\overline{\sqrt{c_{2}}}=\beta+i \alpha$, então se obtém uma solução chamada de breather, ou de pacote de ondas

$$
\begin{gathered}
B_{\alpha, \beta}(t, x):=2 \sqrt{6} \partial_{x}\left[\arctan \left(\frac{\beta}{\alpha} \frac{\operatorname{sen}(\alpha(x+\delta t))}{\cosh (\beta(x+\gamma t))}\right)\right] \\
\gamma=3 \alpha^{2}-\beta^{2}, \quad \delta=\alpha^{2}-3 \beta^{2}
\end{gathered}
$$

Estes breathers foram descobertos por Wadati em 1973 [22] para o modelo mKdV (16). De fato, pode se comprovar que o modelo mKdV desfocalizante (14) também tem soluções breather, mas eles são singulares em vez dos breathers (21) que são regulares em $\mathbb{R}$. Esses breathers ou pacotes de ondas propagam-se com velocidade $\gamma$ (observe em (21), que ela pode ser $>0$ ou $<0$ dependendo da relação entre os parâmetros $\alpha$ e $\beta$ ), oscilando no tempo, sem dispersar ou desaparecer, e até inclusive colidir elasticamente com outras estruturas ou objetos. Isso quer dizer que os breathers têm boas propriedades de estabilidade, pois pequenas perturbações não os afetam, e permanecem essencialmente como estavam antes da perturbação interagir e atuar sobre a solução (ver na Figura 2 um exemplo de (21) e ler [4] para mais detalhes das propriedades de estabilidade desses breathers).

Apesar da expressão (21) ser, funcionalmente falando, mais complicada que a expressão da onda solitária (10), ela pode ser interpretada de maneira simples, quando o parâmetro $\alpha$ (que leva a informação da oscilação da onda) é bem maior que o parâmetro $\beta$ (que leva a informação da amplitude da onda). Isto é, quando $\alpha \gg \beta$. Sendo assim, podemos fazer um desenvolvimento em (21) para $\beta / \alpha \ll 1$ e obter

$$
B_{\alpha, \beta}(t, x) \approx 2 \sqrt{6} \beta \cos (\alpha(x+\delta t)) \operatorname{sech}(\beta(x+\gamma t))+O\left(\frac{\beta}{\alpha}\right),
$$


que é uma oscilação (a função cosseno) "morando" dentro da função sech, que decai exponencialmente rápido no espaço, o que essencialmente é um pacote de ondas. Matematicamente falando, é uma solução periódica no tempo que está localizada no espaço.

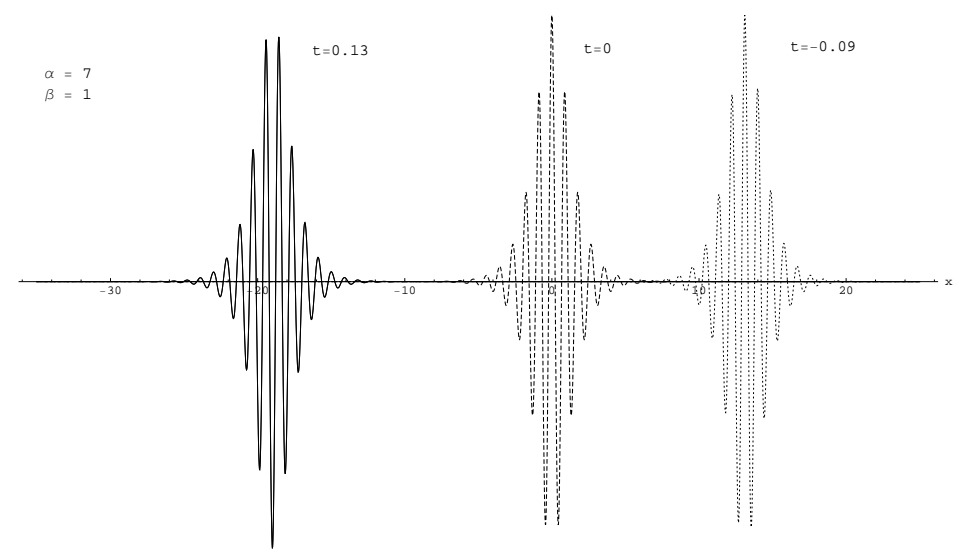

FIgURA 3. Breather de mKdV (16) com scalings $\alpha=$ $7, \beta=1$, evoluindo no tempo.

Da mesma maneira que o sóliton (18) é caracterizado por ser solução da equação diferencial (19), foi provado recentemente em [4] que os breathers também satisfazem uma equação diferencial, sendo sua propriedade principal o fato de ser de quarta ordem. Explicitamente, os breathers de mKdV (16) satisfazem a seguinte equação diferencial (aquí $\left.B_{\alpha, \beta} \equiv B\right)$

$B_{x x x x}-2\left(\beta^{2}-\alpha^{2}\right)\left(B_{x x}+\frac{1}{3} B^{3}\right)+\left(\alpha^{2}+\beta^{2}\right)^{2} B+\frac{5}{3} B B_{x}^{2}+\frac{5}{3} B^{2} B_{x x}+\frac{1}{6} B^{5}=0$.

O fato surpreendente é que outras soluções breather em outros modelos da Física Matemática (Por exemplo sine-Gordon, NLS, Sasa-Satsuma. Ver (32) como exemplo desse fato e [6], [7], [8] e [9] para mais detalhes) satisfazem o mesmo tipo de equação diferencial, isto é, uma equação diferencial de quarta ordem com diferentes termos não lineares do mesmo tipo para os diferentes modelos.

Uma maneira de interpretar esse caráter universal da equação diferencial (23) é observar que usando o operador do sóliton (20), substituindo $Q_{c_{i}}, i=1,2$ pelo breather $B$ e levando em conta que no caso breather $\sqrt{c_{1}}=\overline{\sqrt{c_{2}}}=\beta+i \alpha$, podemos reescrever (23) como

$$
\mathcal{T}_{c_{1}} \mathcal{T}_{c_{2}} B+\frac{1}{3} B^{2} B_{x x}-\frac{1}{3} B B_{x}^{2}+\frac{1}{18} B^{5}=0 .
$$

Portanto, no fundo, essa universalidade da equação diferencial satisfeita por perfis tipo breather não deixa de ser um reflexo do caráter 
"dois-sóliton" do breather, pois pode-se interpretar como se cada sóliton, compondo o estado acoplado breather, fornecesse um operador $\partial_{x}^{2}$ ao operador de quarta ordem da equação diferencial (23). Para mais detalhes sobre essa questão veja [4].

Um aspecto positivo da mKdV (16), e outros modelos da Física Matemática, é que se pode construir soluções que sejam multipacotes de ondas, ou $N$ breathers, ou até breathers periodicamente distribuídos. É por isso que esses modelos são chamados de integráveis, pois de alguma maneira permitem integrar as equações diferenciais para obter as suas soluções. Por exemplo, no caso particular da equação de Gardner [5], podem se obter soluções tipo breather periódico como na Figura 4

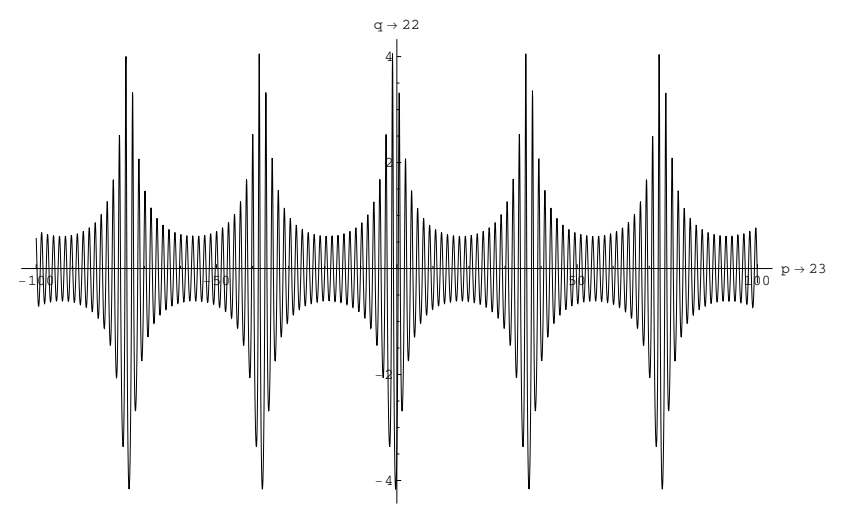

FIgURA 4. Breather espacialmente periódico de Gardner (17).

O leitor poderia se perguntar por que foi preciso ir até a equação mKdV focalizante (16) ou até a equação de Gardner (17) para encontrar estes breathers ou pacotes de ondas, sendo que o primeiro modelo, e mais simples de todos, é a equação de KdV (7). Essa é uma boa e profunda pergunta que só pôde ser respondida recentemente (2018), num trabalho de Muñoz e Ponce [20], onde eles caracterizaram os modelos que possuem breathers e alguns que não admitem esse tipo de solução, sendo que a KdV (7) está entre esses últimos.

\section{PACOTES COMPlexos}

Até o momento, apresentamos soluções reais de modelos matemáticos baseados em funções de $\mathbb{R}^{2}$ em $\mathbb{R}$. A questão é que a natureza é bem mais diversa, e apresenta soluções complexas, isto é, soluções que são funções de $\mathbb{R}^{2}$ em $\mathbb{C}$. Por exemplo, não temos que ir muito longe para descobrir que no eletromagnetismo, as soluções (os campos elétricos e magnéticos) das equações de Maxwell que modelam e descrevem os fenômenos eletromagnéticos são soluções complexas [13]. Já mais perto 
dos modelos (16) e (17) apresentados, existem versões complexas deles, como a mKdV complexa

$$
u_{t}+u_{x x x}+|u|^{2} u_{x}=0, \quad u(t, x) \in \mathbb{C},
$$

que descreve, por exemplo, propriedades de curvas no plano. A mKdV complexa anterior tem soluções simples da forma

$$
u(t, x):=\sqrt{6} \beta e^{i \alpha(x+\delta t)} \operatorname{sech}(\beta(x+\gamma t)),
$$

com $\gamma, \delta$ como em (21). É certo, porém, que as partes real $(\cos (\alpha(x+$ $\delta t)) \operatorname{sech}(\beta(x+\gamma t)))$ ou imaginária $(\operatorname{sen}(\alpha(x+\delta t)) \operatorname{sech}(\beta(x+\gamma t)))$ de (26) comportam-se como breathers, isto é, como oscilações periódicas no tempo, e localizadas no espaço. Entretanto, é o módulo quadrado de $u$, isto é $|u|^{2}$ o que tem significado físico, e no caso de (26) este é

$$
|u(t, x)|^{2}=6 \beta^{2} \operatorname{sech}^{2}(\beta(x+\gamma t)),
$$

que não oscila (é de fato um sóliton) e portanto não é um breather.

Existem outros modelos complexos bem conhecidos para procurar estas soluções oscilatórias localizadas, como na equação (cúbica) de Schrödinger (ou NLS) desfocalizante

$$
i \psi_{t}+\psi_{x x}-|\psi|^{2} \psi=0 .
$$

Esse modelo, que vem da Mecânica Quântica, descreve a evolução ao longo do tempo, das probabilidades de existência, num ponto do espaço-tempo $(t, x)$, de partículas elementares, tais como os elétrons. Bem, nesse caso da NLS desfocalizante, é possível encontrar soluções tipo degrau contínuo ou kink

$$
\psi(t, x):=\sqrt{2} c e^{-2 i c^{2} t} \tanh (c x), \quad c \in \mathbb{R},
$$

chamada de sóliton escuro na ótica não linear (pois $|\psi|^{2}$ refer-se á intensidade da luz). Portanto, ainda não é o tipo de solução oscilatória localizada que estamos procurando. Assim, se procuram modelos matemáticos onde $|\psi|^{2}$ tenha comportamento oscilatório localizado (tipo breather) por si mesmo.

O exemplo mais direto de um modelo com existência de comportamento oscilatório localizado vem da versão NLS focalizante, isto é

$$
i \psi_{t}+\psi_{x x}+|\psi|^{2} \psi=0 .
$$

Especificamente, pode ser visto que a função seguinte, chamada breather de Kuznetsov-Ma (KM) [15, 17]-[3],

$$
\begin{gathered}
\psi(t, x):=e^{i t}\left[1-\sqrt{2} \beta \frac{\left(\beta^{2} \cos (\alpha t)+i \alpha \operatorname{sen}(\alpha t)\right)}{\alpha \cosh (\beta x)-\sqrt{2} \beta \cos (\alpha t)}\right], \\
\alpha:=(8 c(2 c-1))^{1 / 2}, \quad \beta:=(2(2 c-1))^{1 / 2}, \quad c>\frac{1}{2},
\end{gathered}
$$


é uma solução tipo breather ou pacote de onda complexo da NLS (29). Observe que quando fazemos o limite $c \rightarrow \frac{1}{2}$, o breather complexo (30) reduz-se á solução de Peregrine

$$
\psi_{P}(t, x):=e^{i t}\left(1-\frac{4(1+2 i t)}{1+4 t^{2}+2 x^{2}}\right),
$$

a qual é uma função que decai em espaço e tempo. De fato, a solução de Peregrine anterior é uma candidata para explicar a aparição de ondas extremamente altas no oceano. Voltando ao breather (30), é possivel provar que ele satisfaz a seguinte equação diferencial de quarta ordem (ver [8] para mais detalhes)

$$
\begin{aligned}
\psi_{x x x x}+3 \psi_{x}^{2} \bar{\psi} & +\left(4|\psi|^{2}-3\right) \psi_{x x}+\psi^{2} \bar{\psi}_{x x}+2\left|\psi_{x}\right|^{2} \psi \\
& +\frac{3}{2}\left(|\psi|^{2}-1\right)^{2} \psi-\beta^{2}\left(\psi_{x x}+\left(|\psi|^{2}-1\right) \psi\right)=0 .
\end{aligned}
$$

Para obter (32) (e também (23)), a ideia é construir um funcional de Lyapunov $\mathcal{H}$ que tenha como ponto crítico o breather (30), isto é, que a primeira variação desse funcional seja nula quando ela é avaliada na solução breather de interesse. Resumindo,

$$
\text { encontrar } \mathcal{H}[u] \quad \text { com } \quad \mathcal{H}^{\prime}[\psi]=0 .
$$

Nesse caso, a escolha de um bom funcional de Lypunov $\mathcal{H}$ é a chave para encontrar a equação diferencial que caracteriza os breathers. Para mais detalhes, ver $[4,6,7]$ e [8].

Sobre a equação diferencial (32), o leitor pode comparar com a equação (23) que satisfaz o breather da mKdV focalizante. Observe também que nesses exemplos complexos mostrados ((30) e (31)), o valor imaginário não tem sentido físico por si só e nem pode ser visualizado como pacote de fato, mas o que tem significado real e físico é o módulo quadrado da solução $\psi$, isto é $|\psi|^{2}$, que, sim, é um número em $\mathbb{R}^{+}$. Quando por exemplo, desenhamos o gráfico de $|\psi|^{2}$ sendo $\psi$ dada em (30) ou em (31), obtemos um perfil e comportamento breather como podemos ver na Figura 5 no caso do breather de Kuznetsov-Ma.

\section{MAIS PACOTES?}

Até agora apresentamos soluções em uma dimensão espacial, e é evidente que as ondas verdadeiras moram em duas ou mais dimensões. Mas o estudo de modelos bidimensionais e as suas soluções fica como um problema ainda em aberto e com muitas questões sem resposta.

Além disso, todas as soluções apresentadas são "bem comportadas", isto é, elas têm boas propriedades analíticas, como diferenciabilidade em toda a reta, elas mantêm seu perfil ao longo do tempo, e assim por diante. Existem, porém, outros tipos de equações que possuem soluções 


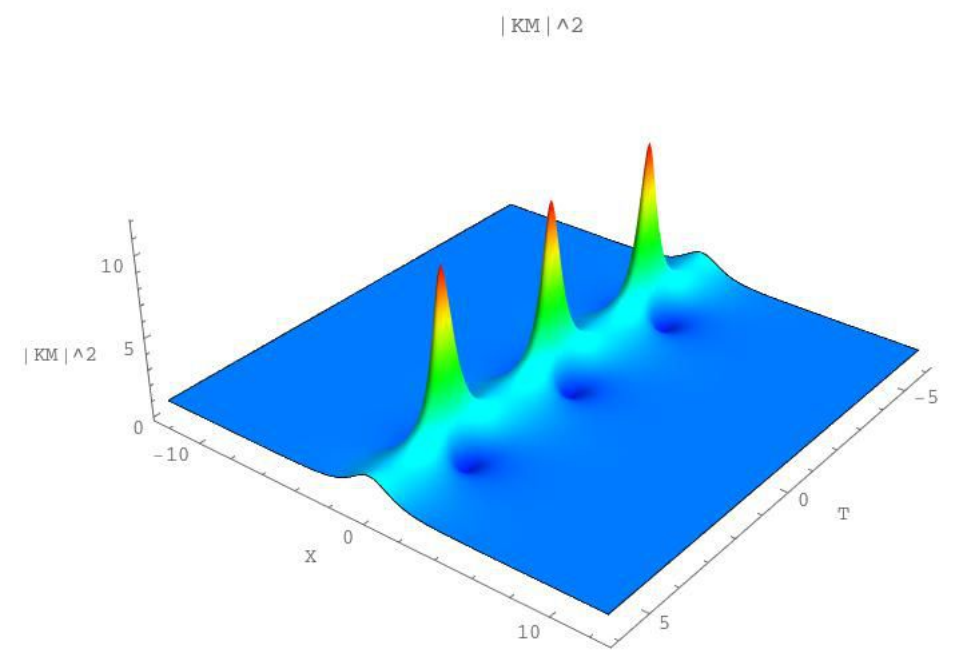

FigurA 5. Breather de Kuznetsov-Ma (30) com $c=0.85$.

mais "estranhas", no sentido que elas perdem suas propriedades de diferenciabilidade ou até geram singularidades (cantos) ou explosão (blow up) em tempo finito, por exemplo. Esses modelos tentam ir um passo mais a frente na descrição dos fenômenos observados na natureza, como ruptura das ondas (quando elas rompem sobre si mesmas) e a aparição no oceano de "extreme ou rogue waves", isto é, ondas de altura extrema que aparecem de maneira súbita ou repentina. A compreensão desses e outros fenômenos pode ajudar a prever eventos extremos e a estabelecer medidas antecipadas de proteção.

\section{REFERÊNCIAS}

[1] M.J. Ablowitz e H. Segur, Solitons and the Inverse Scattering Transform. SIAM Studies in Applied Mathematics, 1981.

[2] N. Akhmediev, A. Ankiewicz e M. Taki, Waves that appear from nowhere and disappear without a trace, Phys. Lett. A 373 (2009) 675-678.

[3] N. Akhmediev e V.I. Korneev, Modulation instability and periodic solutions of the nonlinear Schrödinger equation. Theor. Math. Phys. 69, 1089-1093 (1986).

[4] M.A. Alejo e C. Muñoz, Nonlinear stability of $m K d V$ breathers, Comm. Math. Phys. (2013), Vol. 324, Issue 1, pp. 233-262.

[5] M.A. Alejo, Nonlinear stability of Gardner breathers, Jour. Diff. Equat. 264, n.2, 1192-1230 (2018).

[6] M.A. Alejo, C. Muñoz e J.M. Palacios, On the Variational Structure of Breather Solutions I: Sine-Gordon case, J. Math. Anal. Appl. Vol.453/2 (2017) pp. 1111-1138. 
[7] M.A. Alejo, C. Muñoz e J.M. Palacios, On the variational structure of Breather Solutions II: periodic mKdV case, Electron. J. Diff. Eqns., Vol. 2017 (2017), No. 56, pp. 1-26.

[8] M.A. Alejo, L. Fanelli e C. Muñoz, Stability and instability of breathers in the U(1) Sasa-Satusuma and Nonlinear Schrödinger models, arXiv:1901.10381.

[9] C. Blank, M. Chirilus-Bruckner, V. Lescarret e G. Schneider. Breather solutions in periodic media, Comm. Math. Phys. 302 (3) p. 815-841 (2011).

[10] J.V. Boussinesq, Essai sur la Theorie des eaux courantes, Memoires presentes par divers savants, l'Acad. des Sci. Inst. Nac. Frances, XXIII (1877), 1-680.

[11] P.G. Drazin, Solitons: An Introduction, Cambridge Univ.Press 1989.

[12] R. Hirota, Exact solution of the modified Korteweg-de Vries equation for multiple collisions of solitons, J. Phys. Soc. Japan, 33, no. 5, 1456-1458 (1972).

[13] J.D. Jackson, Classical Electrodynamics, John Wiley \& Sons. (1975).

[14] D. Korteweg e G. de Vries, On the change of form of long waves advancing in a rectangular canal, and on a new type of long stationary waves, Philosophical Magazine 39 (1895), 422-433.

[15] E. Kuznetsov, Solitons in a parametrically unstable plasma, Sov. Phys. Dokl. 22, 507-508 (1977).

[16] G.L. Lamb, Elements of soliton theory. John Wiley \& Sons, Nueva York, 1980.

[17] Y.C. Ma, The perturbed plane-wave solutions of the cubic Schrödinger equation, Stud. Appl. Math. 60, 43-58 (1979).

[18] J.H. Maddocks e R.L. Sachs, On the stability of KdV multi-solitons, Comm. Pure Appl. Math. 46, 867-901 (1993).

[19] R.M. Miura, The Korteweg-de Vries equation, a survey of results. SIAM Rev., 18 (1976) 412-459.

[20] C. Muñoz e G. Ponce, Breathers and the dynamics of solutions to the KdV type equations, Comm. Math. Phys. 367 (2019), 581-598.

[21] J.S. Russell, Report on waves, Fourteenth meeting of the British Association for the Advancement of Science, 1844.

[22] M. Wadati, The modified Korteweg-de Vries equation. J. Phys. Soc. Japan, 34 (1973) 1289-1296.

[23] http://www.amazonialegal.com.br/textos/Pororoca.htm

Departamento de Matemática. Universidade Federal de Santa Catarina-

UFSC., Campus Trindade, 88040-900. Florianópolis-SC, Brasil

Email address: miguel. alejo@ufsc.br 